\title{
Early and Long-Term Prognosis in Patients With and Without Type 2 Diabetes After Carotid Intervention: A Swedish Nationwide Propensity Score Matched Cohort Study
}

\author{
Alexander Zabala ( $\sim$ alexander.zabala@sll.se) \\ https://orcid.org/0000-0001-5389-1271

\section{Anders Gottsäter} \\ Clinical Research Centre: Lunds universitet Clinical Research Centre \\ Marcus Lind
}

Karolinska Institutet Institutionen for klinisk forskning och utbildning Sodersjukhuset

Göteborgs universitet Institutionen för medicin: Goteborgs universitet Institutionen for medicin

\section{Ann-Marie Svensson}

National Diabes Register, Centre of registers, Gothenburg, Sweden

\section{Björn Eliasson}

Göteborgs universitet Institutionen för medicin: Goteborgs universitet Institutionen for medicin

\section{Rebecka Bertilsson}

Centre of registers in region Västra Götaland, Sweden

\section{Jan Ekelund}

Centre of registers, vastra gotaland, Sweden

\section{Thomas Nyström}

Karolinska Institutet Institutionen for klinisk forskning och utbildning Sodersjukhuset

\section{Magnus Jonsson}

Karolinska Institutet Department of Molecular Medicine and Surgery: Karolinska Institutet Institutionen for molekylar medicin och kirurgi

\section{Research Article}

Keywords: Carotid stenosis, Carotid artery stenting, Carotid endarterectomy, Stroke, Type 2 diabetes

Posted Date: February 23rd, 2021

DOl: https://doi.org/10.21203/rs.3.rs-229125/v1

License: (c) (i) This work is licensed under a Creative Commons Attribution 4.0 International License. Read Full License 
Version of Record: A version of this preprint was published at Cardiovascular Diabetology on April 24th, 2021. See the published version at https://doi.org/10.1186/s12933-021-01282-x. 


\section{Abstract}

Objectives

To investigate early and long-term outcomes after treatment of carotid artery stenosis in patients with type 2 diabetes (T2D) compared to patients without T2D.

\section{Design/Method}

This observational nationwide population-based cohort study investigated all T2D patients treated for carotid stenosis registered in the National Swedish Vascular Surgery and the National Diabetes Registries. Data was collected prospectively for all patients after carotid intervention, during 2009-2015. We estimated crude early (within 30-days) hazard ratios (HRs) risk of stroke and death, and long-term HRs risk, adjusted for confounders with 95\% confidence intervals (Cis), for stroke and death and major adverse cardiovascular events (MACE) by using inverse probability of treatment weighting matching.

Results

A total of 1341 patients with T2D and 4162 patients without T2D were included; $89 \%$ treated for symptomatic carotid stenosis, $96 \%$ with carotid endarterectomy. There was an increased early risk, HRs (95\% Cl), for stroke in T2D patients 1.65 (1.17-2.32), whereas risk for early death 1.00 (0.49-2.04) was similar in both groups. During a median follow-up of 4.3 (T2D) and 4.6 (without T2D), with a maximum of 8.0 years; after propensity score matching there was an increased HRs $(95 \% \mathrm{Cl})$ of stroke $1.27(1.05-$ 1.54) and death 1.27 (1.10-1.47) in T2D patients compared to patients without T2D. Corresponding numbers for MACE were 1.21 (1.08-1.35).

Conclusions

Patients with T2D run an increased risk for stroke, death, and MACE after carotid intervention. They also have an increased perioperative risk for stroke, but not for death.

\section{Background}

The prevalence of diabetes has increased dramatically the past decades all over the world, and type 2 diabetes (T2D) accounts for the majority of the cases. T2D has been associated with an increased risk of cardiovascular complications (1). People with T2D have more than a $50 \%$ relative higher risk for stroke compared to those without diabetes (2).

Stroke represents a heterogeneous group of vascular pathologies where risk factors, such as hypertension, atrial fibrillation, smoking, hyperlipidemia and carotid stenosis, contribute to the increased risk. Carotid stenosis accounts for approximately $10-15 \%$ of all ischemic strokes (3). Ever since the landmark studies, i.e. the North American Symptomatic Carotid Endarterectomy Trial (NASCET) (4) and 
the European Carotid Surgery Trial (ECST) (5), carotid endarterectomy has been a cornerstone in reducing the risk of stroke in patients with symptomatic carotid stenosis.

Individuals with diabetes have an increased risk of mortality after surgical revascularization of the coronary arteries (4) demonstrated by several trials (5). Today no trial has investigated the risk of stroke and death in patients with diabetes compared to patients without diabetes, after carotid intervention. Also, large observational studies about the relationship between diabetes and long-term outcome of stroke and death after carotid intervention are scarce (6). Since a large proportion of patients undergoing surgery for carotid stenosis have T2D, it is of importance to investigate and evaluate cardiovascular outcomes after such procedures in those patients.

Therefore, we wanted to investigate early risk of stroke and death, and long-term prognosis of stroke, death, and major adverse cardiovascular events (MACE) after carotid intervention in T2D patients compared to patients without T2D, in a nationwide observational cohort study.

\section{Methods}

This observational population-based cohort study investigated all patients who underwent a surgical procedure for carotid stenosis registered in the Swedish National Registry for Vascular Surgery

(Swedvasc). Data was collected prospectively for all patients with symptomatic or asymptomatic carotid stenosis treated with carotid endarterectomy (CEA) or carotid artery stenting (CAS) during 2009 to 2015.

\section{Databases and definitions}

The unique personal identity number assigned to every Swedish citizen was used as the identifier in the records linkage procedure at the Swedish National Board of Health and Welfare. The database was then anonymized according to regulations. Each of the following registers were linked to the Swedvasc register, i.e. the Swedish National Diabetes Register (NDR), the Swedish National Patient Register (NPR), the Swedish Cause of Death Register, the Swedish Prescribed Drug Register (PDR) and the Longitudinal Integration Database for Health Insurance and Job Market Studies Register (LISA) (7).

All patients undergoing vascular surgery in Sweden are registered in Swedvasc, which is a national patient register established in 1994. Type of intervention, i.e. CEA and CAS, together with preoperative data such as: risk factors, type of treatment, complications and reinterventions are registered in Swedvasc and followed up to 30-days. The external validation of carotid interventions in Swedvasc is almost 100\% (8).

The NDR includes more than $94 \%$ of all patients $\geq 18$ years diagnosed with T2D in Sweden. Healthcare providers report continuously directly to the NDR or via electronic patient records from routine clinical practice. The registry includes data on clinical characteristics, diabetes treatment, risk factors, and diabetes-related complications. T2D was defined according to epidemiological criteria: that is, treatment 
with oral glucose-lowering treatment combined with diet, or diet only; or individuals aged $\geq 40$ years at the time of diagnosis treated with glucose-lowering treatment combined with insulin, or insulin only (9).

In this study, all patients registered in Swedvasc after surgery for carotid stenosis between 2009 and 2015 were identified. Symptomatic carotid stenosis was defined as an ipsilateral acute neurologic event, i.e. stroke or transient ischemic attack within 180 days caused by a carotid stenosis. Asymptomatic carotid stenosis was defined as a carotid stenosis without a prior neurologic event, or neurologic event more than 180-days ago. All registered patients with a defined carotid stenosis were then associated with the registration in NDR and defined as T2D patients and compared with those without T2D diabetes.

\section{Baseline demographic data}

Demographic characteristics were obtained from Swedvasc, however when data was missing in Swedvasc, NDR was used to complement. Information about preexisting history of comorbidities were retrieved from IPR and NDR including: cardiovascular disease, stroke, myocardial infarction, coronary heart disease, heart failure, atrial fibrillation, kidney disease, cancer disease, gastric bypass, psychiatric disorder, and dementia.

Use of pharmacology drug treatment such as: anticoagulation therapy, acetylsalicylic acid, antihypertensive, lipid-lowering, and glucose-lowering treatment, were retrieved from PDR, and was defined as three filled, prescriptions for medication for one year prior to index date, to indicate actual usage.

The LISA register was used to obtain socioeconomic information such as: educational level, marital status, household disposable income and country of birth.

\section{Outcome and follow-up}

Since Swedvasc only provides 30-days outcome after the procedure, the IPR was used to evaluate longterm outcomes. The study population was followed from first carotid interventional procedure (defining the index date) until outcome in the form of: stroke, death, cardiovascular death, MACE, i.e. non-fatal MI, non-fatal stroke and cardiovascular death, or end of study, i.e., December 31, 2017. Only the first hospital admission was used for patients with several of the outcome episodes.

The Cause of Death Register was used to obtain the dates and causes of death. For the cardiovascular death outcome, the following ICD 10 codes were used: I20-I25, I61-I64, R57.0 and R96. ICD 10 codes were used to identify all strokes: 163 and 164 were used for ischemic stroke, 161 to 162 were used for hemorrhagic stroke. Subarachnoid hemorrhage (ICD 10 code I60) was not included. Myocardial infarction was defined by ICD 10 code I21, and heart failure by ICD 10 code I50.

Only the first hospital admission was used for participants with several stroke episodes. Since Swedvasc is a code-based register, information on stroke laterality was not possible to determine. The validity of the diagnoses of stroke, myocardial infarction and heart failure in NPR has previously been evaluated with positive predictive values between $85-95 \%$ (10). 


\section{Statistical analysis}

Descriptive statistics are presented using mean, median, standard deviation (SD), interquartile range (IQR), counts, and percentages with $95 \%$ confidence intervals $(\mathrm{Cl})$ according to variable type. The degree of similarity between T2D patients and patients without T2D is described using the standardized mean difference. Cumulative incidences of all-cause mortality, cardiovascular death, stroke, and MACE are described using Kaplan-Meier curves with lifetables transformed to estimate distribution function rather than survival function. P-values $<0.05$ were considered significant

Outcomes were compared between patients with and without T2D at the end of follow-up using both unadjusted Cox-regression and Inverse Probability of Treatment Weighting (IPTW), i.e., propensity score matching, adjusted Cox-regression and results are presented as hazard ratios (HRs) with associated 95\% $\mathrm{Cl}$. Propensity scores were estimated adjusted for the following variables; age, sex, smoking, indication (asymptomatic/symptomatic), type of intervention (CEA/CAS), lipid lowering treatment, antihypertensive treatment, acetylsalicylic acid, clopidogrel, other oral anticoagulant, ACE-inhibitors, angiotensin receptor blockers, beta blocker, calcium channel blocker, disposable income, education, marital status, country of origin, acute myocardial infarct, coronary heart disease, stroke, cardiovascular disease, atrial fibrillation, heart failure, renal disease, cancer, psychiatric disorder, coronary revascularisation, dementia, gastric bypass, and peripheral arterial disease (Table S1). When estimating propensity score for comparisons within sub-groups, indication and type of surgery were excluded as appropriate. Propensity scores were estimated using a generalized boosted multinomial regression model with an interaction depth of three, a maximum of 10.000 trees, and a shrinkage of 0.01 . The optimal number of trees were selected using a stopping rule applied to the degree of balance.

Most variables were derived from mandatory health data or population registries and therefore have virtually no missing values, except smoking for which $16 \%$ of data was missing. The gradient boosting machine model used in the estimation of the propensity score treats missing values as a separate category and attempts to balance the proportion of missing values as well as non-missing values. Analyses were performed using R 3.4.3.

\section{Results}

\section{Study population and demographic characteristics}

A total of 5503 patients were registered in Swedvasc during the period Jan $1^{\text {st }}, 2009$ - Dec $31^{\text {st }}, 2015$ with a carotid intervention. Out of these 5503 patients 1341 patients (24.4\%) had T2D. The majority of the patients were treated for symptomatic CAS (88.6\% patients without T2D vs. 89\% T2D patients) and CEA was the most common intervention (95.9\% patients without T2D vs. 94.6\% T2D patients).

All baseline demographic characteristics of the patients studied are presented in Table 1. Mean age was 72 (SD 8) years in both groups, with a larger proportion of females among patients without T2D (33.0\%) 
compared to T2D patients (28.2\%). The majority of the patients were treated for a high-grade stenosis (70-99\% NASCET), a small proportion of patients with repeated symptoms were treated for a stenosis of less than $50 \%, 6 \%$ of the patients had a contralateral stenosis in both groups. Patients without T2D were more likely to smoke compared to T2D patients ( $25.8 \%$ vs. $20.1 \%)$. In contrast, it was more common that T2D patients had a history of cardiovascular comorbidities compared to patients without T2D (Table 1). Lipid lowering drugs were used by $79.1 \%$ of the T2D patients, and by $63.0 \%$ among patients without T2D. Also, antiplatelet drugs were more frequently used in T2D patients; acetylsalicylic acid $66.9 \%$ vs. $60.5 \%$, and clopidogrel $21.8 \%$ vs. $18.2 \%$, respectively. Patients without T2D had a higher education level and an increased socioeconomic level than T2D patients (Table 1). There were no differences in the degree of ipsilateral or contralateral degree of carotid stenosis between groups (Table 1). Glycemic control and diabetes duration in T2D patients were HbA1c 55.6 (SD 14.2) $\mathrm{mmol} / \mathrm{mol}(\mathrm{HbA1c} 7.2 \%)$ and 10.5 (SD 7.9) years, respectively.

Table 1. Baseline characteristics of individuals (without 2 diabetes [Non-T2D] vs. type 2 diabetes [T2D]) undergoing carotid intervention. 


\begin{tabular}{|c|c|c|c|c|}
\hline & Non-T2D & T2D & P-value & SMD \\
\hline $\mathrm{n}$ & 4162 & 1341 & & \\
\hline Age, mean (SD) & $72.21(8.30)$ & $72.42(7.72)$ & 0.416 & 0.026 \\
\hline Female, n (\%) & $1372(33.0)$ & $378(28.2)$ & 0.010 & 0.104 \\
\hline Smoking, n (\%) & $875(25.8)$ & $248(20.1)$ & $<0.001$ & 0.134 \\
\hline \multicolumn{5}{|l|}{ History of comorbidities, n (\%) } \\
\hline Cardiovascular disease (\%) & $2265(54.4)$ & $851(63.5)$ & $<0.001$ & 0.185 \\
\hline Stroke & 1989 (47.8) & $743(55.4)$ & $<0.001$ & 0.153 \\
\hline Myocardial infarction & $483(11.6)$ & $243(18.1)$ & $<0.001$ & 0.184 \\
\hline Coronary heart disease & $1119(26.9)$ & $539(40.2)$ & $<0.001$ & 0.285 \\
\hline Heart failure & $243(5.8)$ & $138(10.3)$ & $<0.001$ & 0.164 \\
\hline Atrial fibrillation & $460(11.1)$ & $216(16.1)$ & $<0.001$ & 0.148 \\
\hline Kidney disease & $150(3.6)$ & $86(6.4)$ & $<0.001$ & 0.129 \\
\hline Cancer disease & $450(10.8)$ & $137(10.2)$ & 0.573 & 0.019 \\
\hline Gastric bypass & $2(0.0)$ & $2(0.1)$ & 0.541 & 0.032 \\
\hline Psychiatric disorder & $156(3.7)$ & $41(3.1)$ & 0.272 & 0.038 \\
\hline Dementia & $17(0.4)$ & $5(0.4)$ & 1.00 & 0.006 \\
\hline Degree of ipsilateral carotid stenosis, $\mathrm{n}(\%)^{*}$ & & & 0.52 & 0.036 \\
\hline$\leq 50 \%$ & $229(5.5)$ & $82(6.1)$ & & \\
\hline $50-69 \%$ & $1194(28.7)$ & $397(29.6)$ & & \\
\hline $70-99 \%$ & $2737(65.8)$ & $862(64.3)$ & & \\
\hline Degree of contralateral carotid stenosis, $\mathrm{n}(\%)^{*}$ & & & 0.19 & 0.068 \\
\hline$\leq 50 \%$ & $3105(74.7)$ & $966(72.0)$ & & \\
\hline $50-69 \%$ & $454(10.9)$ & $163(12.2)$ & & \\
\hline $70-99 \%$ & $349(8.4)$ & $132(9.8)$ & & \\
\hline Occlusion & $251(6.0)$ & $80(6.0)$ & & \\
\hline Symptomatic stenosis, n (\%) & $3686(88.6)$ & $1194(89.0)$ & 0.669 & 0.015 \\
\hline Carotid endarterectomy, n (\%) & $3993(95.9)$ & $1269(94.6)$ & 0.050 & 0.062 \\
\hline Peripheral arterial disease (\%) & $175(4.2)$ & $101(7.5)$ & $<0.001$ & 0.142 \\
\hline
\end{tabular}




\begin{tabular}{|c|c|c|c|c|}
\hline \multicolumn{5}{|l|}{ Medication, n (\%) } \\
\hline Lipid lowering drug & $2623(63.0)$ & $1061(79.1)$ & $<0.001$ & 0.361 \\
\hline Antihypertensive drug & $3192(76.7)$ & $1222(91.1)$ & $<0.001$ & 0.401 \\
\hline ACE inhibitor & $1154(27.7)$ & $533(39.7)$ & $<0.001$ & 0.256 \\
\hline Angiotensin II receptor blocker & $755(18.1)$ & $331(24.7)$ & $<0.001$ & 0.160 \\
\hline Beta blocker & $1740(41.8)$ & 777 (57.9) & $<0.001$ & 0.327 \\
\hline Calcium chanel blocker & $1379(33.1)$ & $640(47.7)$ & $<0.001$ & 0.301 \\
\hline Anticoagulant therapy ${ }^{\dagger}$ & $1434(34.5)$ & $542(40.4)$ & $<0.001$ & 0.123 \\
\hline Acetylsalicylic acid & $2517(60.5)$ & $897(66.9)$ & $<0.001$ & 0.134 \\
\hline P2Y12 inhibitor (Clopidogrel) & $757(18.2)$ & $293(21.8)$ & 0.003 & 0.092 \\
\hline \multicolumn{5}{|l|}{ Antihyperglycaemic agent, $n(\%)$} \\
\hline Metformin & $0(0.0)$ & $744(55.5)$ & $<0.001$ & 1.579 \\
\hline Sodium-glucose-transport-2 inhibitor & $0(0.0)$ & $4(0.3)$ & $<0.001$ & 0.077 \\
\hline Incretin ${ }^{\ddagger}$ & $0(0.0)$ & $74(5.5)$ & $<0.001$ & 0.342 \\
\hline Insulin & $0(0.0)$ & $492(36.7)$ & $<0.001$ & 1.077 \\
\hline Disposable income per month after tax, USD & $2078.3(2609)$ & $1875.8(204)$ & 0.009 & 0.086 \\
\hline Educational level, n (\%) & & & 0.012 & 0.096 \\
\hline Compulsory school & $1658(40.3)$ & $567(42.8)$ & & \\
\hline Upper secondary & $1707(41.5)$ & $562(42.4)$ & & \\
\hline College/University & $753(18.3)$ & $196(14.8)$ & & \\
\hline Civil status, n (\%) & & & 0.092 & 0.079 \\
\hline Single & $385(9.3)$ & $132(9.9)$ & & \\
\hline Married & $2294(55.2)$ & $712(53.1)$ & & \\
\hline Divorced & $822(19.8)$ & $303(22.6)$ & & \\
\hline Widowed & $657(15.8)$ & $193(14.4)$ & & \\
\hline Origin, $\mathrm{n}(\%)$ & & & 0.015 & 0.088 \\
\hline Sweden & $3574(85.9)$ & $1112(82.9)$ & & \\
\hline Europe except Sweden & $298(7.2)$ & $106(7.9)$ & & \\
\hline Rest of the world & $290(7.0)$ & $123(9.2)$ & & \\
\hline
\end{tabular}


*Definition accordingly to The North American Symptomatic Carotid Endarterectomy Trial. ${ }^{\dagger}$ Anticoagulant therapy

includes Heparin, Low molecular Heparin, Non-Vitamin $\mathrm{K}$ antagonist and Vitamin $\mathrm{K}$ antagonists. ${ }^{\ddagger}$ Incretin, includes

dipeptidyl peptidase-4 inhibitors and glucagon-like peptide-1. SMD, Standardised mean difference. SD, Standard deviation.

Categorical variables are presented as number (\%) and continuous variables are presented as mean (SD).

\section{Early (within 30-days) complication rates}

The perioperative (within 30-days) complication rate for stroke was 3.7\% (95\% $\mathrm{Cl} 2.7 \%$ - $4.7 \%$ ) compared to $2.3 \%(95 \% \mathrm{Cl} 1.8 \%-2.7 \%)$ for patients without T2D. There was no difference in rates of perioperative death between groups: T2D patients $0.7 \%$ (95\% $\mathrm{Cl} 0.5 \%-1.0 \%)$ vs. patients without T2D $0.7 \%(95 \% \mathrm{Cl}$ $0.3 \%-1.2 \%)$, respectively. Corresponding early crude HRs $(95 \% \mathrm{Cl})$ for stroke was $1.65(1.17-2.32)$ in T2D patients compared to patients without T2D, whereas early crude risk for death was similar $1.00(0.49$ $2.04)$ in both groups.

\section{Long-term outcome events and crude incidence rate between groups after carotid intervention}

Median follow up time 4.3 (IQR 2.9-6.0) years for T2D patients and 4.6 (IQR 3.1-6.3) years for patients without T2D with a maximum follow-up time of 8.0 years (both groups). Kaplan-Meier estimated survival curves demonstrating the cumulative number of patients at risk and the proportions for the outcomes, i.e., stroke, death, and MACE are shown in Figure 1A-C. Numbers of event and crude incidence rate for the studied outcomes were all increased in T2D patients compared to patients without T2D (Table 2).

Table 2. Total number of events and incidence rate with exact $95 \%$ Poisson confidence interval for the outcomes of individuals without type 2 diabetes (Non-T2D) and individuals with type 2 diabetes (T2D), respectively, undergoing carotid intervention. 


\begin{tabular}{|c|c|c|c|c|}
\hline & \multicolumn{2}{|c|}{$\begin{array}{l}\text { Non-T2D } \\
(n=4162)\end{array}$} & \multicolumn{2}{|c|}{$\begin{array}{l}\text { T2D } \\
(n=1341)\end{array}$} \\
\hline Outcome & $\begin{array}{l}\text { Events } \\
\text { (n) }\end{array}$ & $\begin{array}{l}\text { Incidence rate } \\
\text { (per } 1000 \text { patients) }\end{array}$ & $\begin{array}{l}\text { Events } \\
\text { (n) }\end{array}$ & $\begin{array}{l}\text { Incidence rate } \\
\text { (per } 1000 \text { patients) }\end{array}$ \\
\hline Stroke & 453 & $25.1(22.8-27.5)$ & 193 & $35.7(30.8-41.1)$ \\
\hline Ischemic stroke & 380 & $20.8(18.8-23.0)$ & 166 & $30.3(25.9-35.3)$ \\
\hline Hemorrhagic stroke & 68 & $3.5(2.7-4.5)$ & 20 & $3.4(2.1-5.2)$ \\
\hline Death & 725 & $37.4(34.7-40.2)$ & 324 & $54.5(48.7-60.7)$ \\
\hline Cardiovascular death & 161 & $8.3(7.1-9.7)$ & 89 & $15.0(12.0-18.4)$ \\
\hline MACE & 1248 & $82.7(78.2-87.4)$ & 551 & $135(123.4-146.1)$ \\
\hline
\end{tabular}

HR, Hazard Ratio; MACE, Major adverse cardiovascular events, i.e. Non-fatal myocardial infarction, nonfatal stroke and cardiovascular death.

\section{Propensity score matched risk of long-term outcomes between groups after carotid intervention}

After propensity score matching the groups were well balanced concerning all covariates and demographic characteristics (Table S2). HRs $(95 \% \mathrm{Cl})$ for stroke, death, cardiovascular death, and MACE were all increased in patients with T2D: 1.27 (1.05-1.54), 1.27 (1.10-1.47), 1.60 (1.20-2.12), and 1.21 (1.08-1.35), respectively, compared to patients without T2D (Table 3).

Table 3. Cox-regression estimates (crude and after adjustments using inverse probability of treatment weighting [IPTW]) for individuals with type 2 diabetes compared with individuals without type 2 diabetes with asymptomatic and symptomatic carotid stenosis undergoing any carotid intervention. 


\begin{tabular}{|c|c|c|c|c|}
\hline & Crude & & IPTW adjustment ${ }^{*}$ & \\
\hline Outcome & $\mathrm{HR}(95 \% \mathrm{Cl})$ & P-value & $\mathrm{HR}(95 \% \mathrm{Cl})$ & P-value \\
\hline Stroke & $1.38(1.17$ - 1.64) & $<0.001$ & $1.27(1.05-1.54)$ & 0.012 \\
\hline Ischemic stroke & $1.41(1.18-1.69)$ & $<0.001$ & $1.30(1.06-1.60)$ & 0.011 \\
\hline Hemorrhagic stroke & $0.95(0.58-1.56)$ & 0.834 & $1.29(1.06-1.55)$ & 0.009 \\
\hline Death & $1.48(1.30-1.68)$ & $<0.001$ & $1.27(1.10-1.47)$ & 0.001 \\
\hline Cardiovascular death & $1.79(1.38-2.32)$ & $<0.001$ & $1.60(1.20-2.12)$ & 0.001 \\
\hline MACE & $1.52(1.37-1.68)$ & $<0.001$ & $1.21(1.08-1.35)$ & 0.001 \\
\hline
\end{tabular}

$\mathrm{Cl}$, Confidence interval. HR, Hazard Ratio; MACE, Major adverse cardiovascular events. i.e. Non-fatal myocardial infarction, non-fatal stroke and cardiovascular death. IPTW, Inverse probability of treatment weighting. ${ }^{*}$ Adjusted for all variables in Table S1 (Supplemental Material).

The corresponding numbers of the HRs for the outcomes, after dividing groups in symptomatic vs. asymptomatic carotid stenosis, and CEA vs. CAS procedures, are shown in Table S3 and Table S4, respectively. The proportion of events for CAS and asymptomatic stenosis compared to CEA and symptomatic stenosis were small (Table S3 and Table S4)

\section{Discussion}

In this large nationwide cohort of patients that underwent carotid surgery in Sweden during a follow-up period of 8 years, we observed that patients with T2D had an increased risk of stroke and death compared to patients without T2D after carotid intervention. Perioperatively there was an increased risk of stroke, but not for death, in T2D patients.

In previous studies the difference in perioperative complications between patients with or without diabetes has mainly been attributed to mortality $(6,11)$. This is in contrast with the current observational study in which rates of perioperative death were similar between groups after carotid intervention. Even though there was an increased perioperatively risk of stroke for T2D patients, the risk was below the recommended threshold of $6 \%$ to justify carotid intervention (12).

In the present study long-term risk of stroke, death, and cardiovascular events (MACE) were all increased in T2D patients compared to patients without T2D. This contradicts a recent observational study that demonstrated increased perioperative mortality in the presence of diabetes after carotid surgery, a difference that was negligible during follow-up (6). Other studies report different results regarding prognosis; some have reported that patients with diabetes are not at an increased risk (6), while others 
support the role of diabetes as an increased risk factor for stroke and death after carotid intervention (12). Increased long-term mortality after carotid intervention in diabetic patients compared to patients without diabetes was recently demonstrated in a meta-analysis comprising more than 4000 patients with diabetes (13). However, since a minority of the studies included in the meta-analysis only reported mortality; long-term prognosis of stroke or cardiovascular events after carotid intervention in patients with diabetes is still to a large extent unknown (13).

Diabetes is a well-known risk factor for adverse cardiovascular events, and must be paid attention to, when choosing type of revascularization in multivessel coronary artery disease as certain procedures, i.e. coronary artery bypass grafting, is superior to others (14). Patients with diabetes have an accelerated and more extensive coronary atherosclerosis than patients without diabetes (15). In carotid arteries, studies demonstrating signs of pronounced atherosclerosis in patients with diabetes compared to patients without diabetes (16). Already in children with T2D, carotid intima-media thickness is increased (17), which in turn is associated with future cardiovascular events (18). Strong cardiovascular risk factors such as smoking, hypertension, and dyslipidaemia are well established contributing risk factors for carotid stenosis. In insulin resistance, more or less obligate in T2D patients, some strong cardiovascular risk factors are concomitantly represented, i.e., hypertension, obesity, dyslipidaemia, kidney failure, and dysglycemia(19), which all can have contributed to the increased risk of stroke and death and MACE after carotid surgery observed in the current study. Interestingly, insulin resistance per se is associated with increased intima media thickness in diabetes (9). In the present study, only smoking status was collected in the Swedvasc register and subsequently adjusted for between groups, whereas the other above mentioned metabolic cardiovascular risk factors were not compared between groups which may all have contributed to the increased cardiovascular risk after carotid intervention observed in T2D patients.

Not only metabolic factors can contribute to an increased risk of cardiovascular events after carotid intervention, co-morbidities and medications for reducing cardiovascular risk are also relevant. In the present study, at baseline cardiovascular comorbidities and medications such as lipid-lowering and antiplatelets agents were more often used in T2D patients. However, after propensity score matching groups were equal to all risk factors of interest that could have influenced our cardiovascular outcomes of interest including death in the long-term follow up. The outcomes of interest in our study are of high external validity (10) and by using the NDR we can be confident that the classification of T2D was correct This is in contrast with other observational studies $(20,22)$, in which the discrimination between different types of diabetes was not accurate.

Our study showed almost a $30 \%$ relative long-term increased risk of stroke in T2D patients compared to patients without T2D after carotid intervention. Covariates involved in the associations of the outcome of interest at follow-up were all well balanced due to the propensity score matching and therefore cannot explain the higher risk of stroke and death observed in patients with T2D. The net benefit from carotid intervention depends on the complication rate as well as the risk of stroke during follow up, theoretically patients with T2D may even benefit more (relatively) if undergoing carotid intervention than patients without T2D. In the present study, the cumulative risk of stroke during five-years follow-up in the present 
study was for T2D patients $16 \%$ vs. $12 \%$ in non-T2D patients. This could be compared to the five years cumulative risk of any stroke event of $24 \%$ in the surgical arm of the NASCET trial (20). This demonstrates that patients undergoing carotid intervention due to a severe symptomatic carotid stenosis are at high risk for long-term complications such as stroke and death. Despite this, the benefit of CEA in patients with symptomatic carotid stenosis is durable and convincing in comparison with medical treatment alone (21).

CEA has been shown to reduce the risk of stroke in patients with symptomatic carotid stenosis $(20,22-$ 24), whereas carotid artery stenting has evolved as an alternative minimal invasive treatment. Several randomised controlled trials have shown that CAS is associated with a higher proportion of perioperative stroke or death as compared to CEA (25), therefore the net benefit from the carotid intervention may also depend on the procedure chosen. Moreover, carotid surgery is also proven beneficial in patients with asymptomatic carotid stenosis $(23,26)$. Even though the present observational study was large, the subgroups of patients undergoing CEA or CAS for symptomatic or asymptomatic stenosis were small and therefore results in these are less reliable.

The strengths of the present observational nationwide study were its size and the high external validity of the registries used for the outcomes of interest. The Cause of Death register used to retrieve mortality data has complete coverage of the country, and therefore we had no loss to follow-up. We used propensity score matching thereby limiting confounding factors for the results. However, our study has limitations. There is a lack of information about the type, severity, and laterality of the stroke. Also, there is a lack of information about metabolic cardiovascular risk factors such as blood pressure, blood lipids, and kidney function, i.e., estimated glomerular filtration rate which might have affected our results. It is however known that patients with diabetes are more exposed to these cardiovascular risk factors compared to patients without diabetes. Even though the comparison of the groups was made before and after propensity score matching, thereby limiting the influence of confounding factors on the results, we cannot entirely rule out some unmeasured differences between the groups which may have affected the results.

\section{Conclusions}

This nationwide, propensity score matched, cohort study shows that patients with T2D have an increased long-term risk for stroke and death and MACE, compared to patients without T2D, after carotid intervention. There was also an increased risk of preoperative stroke, but not death, in T2D patients compared to patients without T2D. Even though the periprocedural complications after carotid intervention today is low and acceptable, our finding highlights the importance to define certain risk groups such as diabetes.

\section{Declarations}

\section{Ethical approval and consent to partcipate}


All patients have consented to being reported in the Swedvasc and NDR. No individual consent is required for inclusion in this study, according to Swedish law. The study was approved by the ethics committee of the University of Lund, Sweden (2016/232 and 2016/544).

\section{Consent for publication}

Not applicable

\section{Availability of data and materials}

The datasets analysed during the current study are available from the corresponding author on reasonable request.

\section{Competing interests}

TN has received unrestricted grants from AstraZeneca and NovoNordisk and has served on national advisory boards of Amgen, Novo Nordisk, Sanofi-Aventis, Eli Lilly, MSD and Boehringer Ingelheim. AG has served on national advisory boards of Amgen, Astra Zeneca, Bayer, Boehringer-Ingelheim, Bristol Myers Squibb, Gore, Leo Pharma, Novartis, Novo Nordisk, Pfizer, and Sanofi. BE reports personal fees from AMGEN, AstraZeneca, Boehringer Ingelheim, Eli Lilly, Merck Sharp \& Dohme, Mundipharma, Navamedic, NovoNordisk, RLS Global, and grants and personal fees from Aanofi, all outside the submitted work. ML has received research grants from DexCom and Novonordisk and been consultant for Astra Zeneca, Boheringer Ingelheim, DexCom, Eli Lilly, MSD and Novonordisk.

\section{Funding}

TN is funded by the Hjärt-Lungfonden (grant 20190298) and ALF agreement between Stockholms Läns Landsting and Karolinska Institutet (grant 20170120). AG is funded by grants from Research Funds at Skåne University Hospital, Region Skåne (430751), the Hulda Ahlmroth Foundation, and from the Swedish Government under the LUA/ALF agreement.

\section{Author Contribution}

All authors participated in the research design. JE and RB performed the data management and statistical analyses after discussion with all authors. All authors participated in data interpretation. AZ, $\mathrm{MJ}$ and TN wrote the first draft of the manuscript, and all authors took final responsibility for the decision to submit for publication.

\section{Acknowledgements}

The Swedvasc steering committee: Katarina Björses, Lena Blomgren, Magnus Jonsson, Kevin Mani, Mari Holsti, Kristian Smidfelt.

\section{References}


1. Rawshani A, Rawshani A, Franzén S, Sattar N, Eliasson B, Svensson A-M, et al. Risk Factors, Mortality, and Cardiovascular Outcomes in Patients with Type 2 Diabetes. N Engl J Med. 2018 Aug 16;379(7):633-44.

2. Zabala A, Darsalia V, Holzmann MJ, Franzén S, Svensson A-M, Eliasson B, et al. Risk of first stroke in people with type 2 diabetes and its relation to glycaemic control: A nationwide observational study. Diabetes Obes Metab. 2020;22(2):182-90.

3. Marulanda-Londoño E, Chaturvedi S. Stroke due to large vessel atherosclerosis. Neurol Clin Pract. 2016 Jun;6(3):252-8.

4. Nyström T, Holzmann MJ, Eliasson B, Svensson A-M, Kuhl J, Sartipy U. Estimated glucose disposal rate and long-term survival in type 2 diabetes after coronary artery bypass grafting. Heart Vessels. 2017 Mar 1;32(3):269-78.

5. Verma S, Farkouh ME, Yanagawa B, Fitchett DH, Ahsan MR, Ruel M, et al. Comparison of coronary artery bypass surgery and percutaneous coronary intervention in patients with diabetes: a metaanalysis of randomised controlled trials. Lancet Diabetes Endocrinol. 2013 Dec 1;1(4):317-28.

6. Dorigo W, Pulli R, Pratesi G, Fargion A, Marek J, Innocenti AA, et al. Early and long-term results of carotid endarterectomy in diabetic patients. J Vasc Surg. 2011 Jan 1;53(1):44-52.

7. Ludvigsson JF, Svedberg P, Olén O, Bruze G, Neovius M. The longitudinal integrated database for health insurance and labour market studies (LISA) and its use in medical research. Eur $J$ Epidemiol. 2019 Apr 1;34(4):423-37.

8. Venermo M, Lees T. International Vascunet Validation of the Swedvasc Registry. Eur J Vasc Endovasc Surg. 2015 Dec 1;50(6):802-8.

9. Rathsman B, Rosfors S, Sjöholm A, Nyström T. Early signs of atherosclerosis are associated with insulin resistance in non-obese adolescent and young adults with type 1 diabetes. Cardiovasc Diabetol. 2012 Nov 27;11:145.

10. Ludvigsson JF, Andersson E, Ekbom A, Feychting M, Kim J-L, Reuterwall C, et al. External review and validation of the Swedish national inpatient register. BMC Public Health. 2011 Jun 9;11:450.

11. Ahari A, Bergqvist D, Troeng T, Elfstrom J, Hedberg B, Ljungstrom K, et al. Diabetes Mellitus as a Risk Factor for Early Outcome After Carotid Endarterectomy - a Population-based Study. Eur J Vasc Endovasc Surg. 1999 Aug;18(2).

12. Naylor AR, Ricco J-B, de Borst GJ, Debus S, de Haro J, Halliday A, et al. Editor's Choice - Management of Atherosclerotic Carotid and Vertebral Artery Disease: 2017 Clinical Practice Guidelines of the European Society for Vascular Surgery (ESVS). Eur J Vasc Endovasc Surg Off J Eur Soc Vasc Surg. 2018 Jan;55(1):3-81. 
13. Hussain MA, Bin-Ayeed SA, Saeed OQ, Verma S, Al-Omran M. Impact of diabetes on carotid artery revascularization. J Vasc Surg. 2016 Apr 1;63(4):1099-1107.e4.

14. A Randomized Trial of Therapies for Type 2 Diabetes and Coronary Artery Disease. N Engl J Med. 2009 Jun 11;360(24):2503-15.

15. Roffi M, Angiolillo DJ, Kappetein AP. Current concepts on coronary revascularization in diabetic patients. Eur Heart J. 2011 Nov 2;32(22):2748-57.

16. Yahagi K, Kolodgie FD, Lutter C, Mori H, Romero ME, Finn AV, et al. Pathology of Human Coronary and Carotid Artery Atherosclerosis and Vascular Calcification in Diabetes Mellitus. Arterioscler Thromb Vasc Biol. 2017 Feb;37(2):191-204.

17. Berenson GS, Srinivasan SR, Bao W, Newman WP, Tracy RE, Wattigney WA. Association between multiple cardiovascular risk factors and atherosclerosis in children and young adults. The Bogalusa Heart Study. N Engl J Med. 1998 Jun 4;338(23):1650-6.

18. Bots ML, Hoes AW, Koudstaal PJ, Hofman A, Grobbee DE. Common carotid intima-media thickness and risk of stroke and myocardial infarction: the Rotterdam Study. Circulation. 1997 Sep 2;96(5):1432-7.

19. Laakso M, Kuusisto J. Insulin resistance and hyperglycaemia in cardiovascular disease development. Nat Rev Endocrinol. 2014 May;10(5):293-302.

20. Ferguson Gary G., Eliasziw Michael, Barr Hugh W. K., Clagett G. Patrick, Barnes Robert W., Wallace M. Christopher, et al. The North American Symptomatic Carotid Endarterectomy Trial. Stroke. 1999 Sep 1;30(9):1751-8.

21. Barnett HJ, Taylor DW, Eliasziw M, Fox AJ, Ferguson GG, Haynes RB, et al. Benefit of carotid endarterectomy in patients with symptomatic moderate or severe stenosis. North American Symptomatic Carotid Endarterectomy Trial Collaborators. N Engl J Med. 1998 Nov 12;339(20):141525 .

22. European Carotid Surgery Trialists' Collaborative Group. Randomised trial of endarterectomy for recently symptomatic carotid stenosis: final results of the MRC European Carotid Surgery Trial (ECST). The Lancet. 1998 May 9;351(9113):1379-87.

23. Walker MD, Marler JR, Goldstein M, Grady PA, Toole JF, Baker WH, et al. Endarterectomy for Asymptomatic Carotid Artery Stenosis. JAMA. 1995 May 10;273(18):1421-8.

24. Rosenfield K, Matsumura JS, Chaturvedi S, Riles T, Ansel GM, Metzger DC, et al. Randomized Trial of Stent versus Surgery for Asymptomatic Carotid Stenosis. N Engl J Med. 2016 Mar 17;374(11):101120. 
25. Batchelder AJ, Saratzis A, Naylor AR. Editor's Choice - Overview of Primary and Secondary Analyses From 20 Randomised Controlled Trials Comparing Carotid Artery Stenting With Carotid Endarterectomy. Eur J Vasc Endovasc Surg. 2019 Oct 1;58(4):479-93.

26. Halliday A, Harrison M, Hayter E, Kong X, Mansfield A, Marro J, et al. 10-year stroke prevention after successful carotid endarterectomy for asymptomatic stenosis (ACST-1): a multicentre randomised trial. The Lancet. 2010 Sep 25;376(9746):1074-84.

\section{Figures}




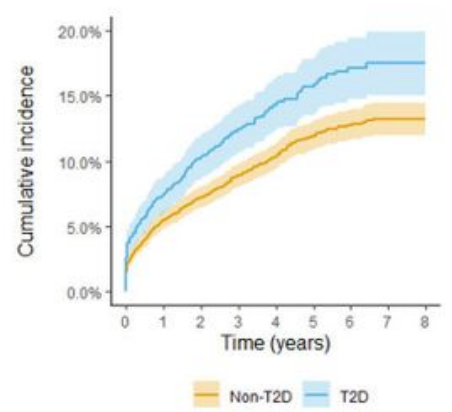

$41623844367629752292 \quad 16601065517 \quad 0$ $\begin{array}{llllllllll}1341 & 1209 & 1128 & 884 & 644 & 472 & 297 & 129 & 0\end{array}$

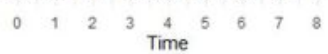

B)

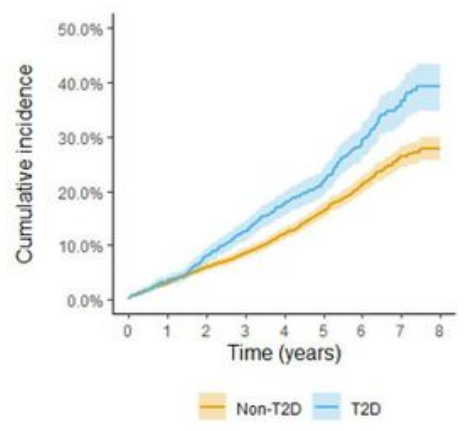

Number at risk

Non-T2D $4162403139133212251918531203 \quad 582 \quad 0$

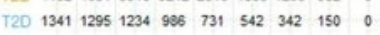

$\begin{array}{lllllllll}0 & 1 & 2 & 3 & 4 & 5 & 6 & 7 & 8\end{array}$

C)

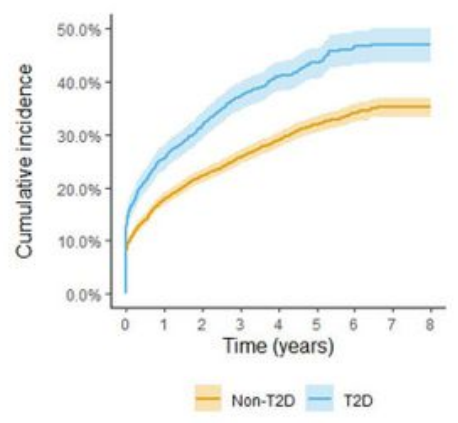

Number at risk

Non-T2D $4162335731092444 \quad 1845 \quad 1292 \quad 800 \quad 385 \quad 0$ $\begin{array}{llllllllll}12 D & 1341 & 983 & 883 & 646 & 448 & 312 & 195 & 75 & 0\end{array}$ $\begin{array}{lllllllll}0 & 1 & 2 & 3 & 4 & 5 & 6 & 7 & 8\end{array}$

\section{Figure 1}

Crude Kaplan-Meier curves demonstrating cumulative incidence and number at risk of A) Stroke B) Death, and C) MACE (major adverse cardiovascular events, i.e. Non-fatal myocardial infarction, non-fatal stroke and cardiovascular death) after carotid interventions (i.e., carotid endarterectomy and carotid artery stenting) among patients without type 2 diabetes (Non-T2D) and patients with type 2 diabetes (T2D). Shaded areas represent $\mathrm{Cl}$ 95\%. 


\section{Supplementary Files}

This is a list of supplementary files associated with this preprint. Click to download.

- SupplementalMaterial210209.docx 